\title{
A Theorem of Local-Torelli Type
}

\author{
D. Lieberman ${ }^{\star}$ and R. Wilsker \\ Brandeis University, Department of Mathematics, Waltham, MA 02154, USA \\ C. Peters \\ Rijksuniversiteit Leiden, Mathematisch Instituut, Wassenaarseweg 80 , Leiden, The Netherlands
}

Given different complex structures on a fixed compact differentiable manifold $X$ the Torelli problem for $X$ is whether these structures are distinguishable at the cohomology level, for example by the position of the subspace $H^{0}\left(X, \Omega^{k}\right) \leqq H^{k}(X, \mathbb{C})$ consisting of classes containing a holomorphic differential $k$-form. Results of this type are classical for Riemann surfaces and complex tori and have been established more recently for various classes of higher dimensional manifolds.

An infinitesimal analog of this problem has been systematically formulated by Griffiths in [2]. Namely, given $X_{s}, s \in S$ a differentiably trivial family of compact Kähler manifolds parametrized by $S$, then $g=\operatorname{dim} H^{0}\left(X_{s}, \Omega^{k}\right)$ is independent of $s$ and one obtains a map $P: S \rightarrow$ Grass $(g, N)$ assigning $s \rightarrow H^{0}\left(X_{s}, \Omega^{k}\right) \subseteq H^{k}\left(X_{s}, \mathbb{C}\right)$. Fixing $s \in S$ and letting $X=X_{s}$, we recall that the differential $\Theta_{s, s} \rightarrow \Theta_{P(s), \text { Grass }}$ is computed by composing the Kodaira-Spencer map $\varrho: \Theta_{s, s} \rightarrow H^{1}\left(X, \Theta_{X}\right)$ with the cup product mapping

$$
\lambda: H^{1}(X, \Theta) \rightarrow \operatorname{Hom}\left(H^{0}\left(X, \Omega^{k}\right), H^{1}\left(X, \Omega^{k-1}\right)\right) .
$$

(One has a natural identification of this Hom with a subspace of $\Theta_{\text {Grass }}$ )

Assuming $S$ is versal, so that $\varrho$ is an isomorphism, one has a problem of local Torelli type to prove that $\lambda$ is injective under suitable hypotheses. In the sequel we assume $k=n=\operatorname{dim} X$ and employ the phrase "the local Torelli theorem holds" or "the $n$-forms give local moduli" to mean that (1.1) is injective. Clearly we must assume that $H^{0}\left(X, \Omega^{n}\right)$ is sufficiently rich, e.g. $H^{0}\left(X, \Omega^{n}\right) \neq 0$, in order to have any hope. In the case $n=1$ it is in fact necessary and sufficient to assume that the canonical map $X \rightarrow \mathbb{P}\left(H^{0}\left(X, \Omega^{1}\right)\right)$ is an imbedding (by a classical and rather difficult theorem of Noether). Conjecturally when the canonical bundle $\Omega_{X}^{n}$ is sufficiently ample, the local Torelli problem will have an affirmative answer. This conjecture has been verified in many special cases, cf. $[2,4,5]$. The investigations of Peters led him to discover a simple proof of

* Supported in part by NSF GP MCS 7611312 
Theorem 1. Let $X$ be a compact Kahler n-manifold $\mathscr{L} \rightarrow X$ a line bundle such that $\mathscr{L}^{\otimes k}=\Omega_{x}^{n}$ for $k>0$ and assume

1) the map $f: X \rightarrow \mathbb{P}^{N}$, given by $H^{0}(X, \mathscr{L})$, is everywhere defined and $\operatorname{dim} f(X)=n$;

2) $H^{0}\left(X, \Omega^{n-1} \otimes \mathscr{L}\right)=0$;

3) given any small deformation $X_{s}, s \in S$, of $X$ there exists $f_{s}: X_{s} \rightarrow \mathbb{P}^{N}$ extending $f$.

Then the position of $H^{0}\left(X, \Omega^{n}\right) \subseteq H^{n}(X, \mathbb{C})$ yields local moduli for $X$, i.e. the map (1.1) is injective.

One may conclude from this theorem the local Torelli results of $[4,5]$.

Corollary 1 (Theorem 5.4 in [4]). The local Torelli theorem holds for complete intersections $X \subseteq \mathbb{P}^{N}$ once $\Omega_{X}^{n}$ is ample.

Proof. Taking $\mathscr{L}=\mathscr{O}(1) / X$, one has $\Omega_{X}^{n}=\mathscr{L}^{\otimes k}$ by adjunction and $k>0$ for ampleness. It suffices to verify 2 (see Theorem 1 ' below), which follows inductively from Bott's vanishing theorem, see Erratum to [4].

Corollary 2 (Theorems 3.1 and 3.4, in [5]). The local Torelli theorem holds for cyclic branched covers $V \rightarrow \mathbb{P}^{N}$ branched along a hypersurface of degree $m n$ where $m>1$; $m(n-1)-N-1>0$; and if $n=2$ then $m \neq 3$. Similarly the result holds for cyclic branched covers of the Hirzebruch surfaces $\Sigma_{r}$ branched along a curve type $(\mu, v)$ with $\mu \geqq r \cdot v, v \geqq 3$.

Proof. Let $\mathscr{L}$ be the pull back of $O(1)$. The condition $\Omega^{n}=\mathscr{L}^{k}$ and $k>0$ is equivalent to $m(n-1)-N-1>0$ (resp. follows from $\mu \geqq r v, v \geqq 3$ ). Conditions 1) and 3) are known to hold under the above assumptions (e.g. [5]). As to Condition 2): In the first case take $k=m(n-1)-N-1$ and one can prove that $H^{0}\left(X, \Omega^{m-1}(1)\right)$ $=H^{0}\left(X, T_{X} \otimes(k+1)=0\right.$ by means of the exact sequences given in 1.8 of [5]. This boils down to another application of Bott's vanishing theorem. In the second case take $k=1$ and $H^{0}\left(X, T_{X} \otimes K_{X}^{2}\right)$ dualizes to $H^{2}\left(X, T_{X}\right)$ which is zero due to 1.8 and 3.2 of $[5]$.

We remark that the theorem as it stands cannot be applied to yield the classic Noether's theorem-for $\mathscr{L}=\Omega_{X}^{1}, X$ a curve, the Conditions 1) and 3 ) are satisfied but 2 requires $H^{0}\left(X, \Omega^{1}\right)=0$ which is too strong. In attempting to weaken Condition 2), Lieberman and Wilsker succeeded in significantly weakening Assumptions 1 and 2 and eliminating 3 :

Theorem $1^{\prime}$. Let $X$ be a compact Kähler n-manifold $\mathscr{L} \rightarrow X$ a line bundle such that $\mathscr{L}^{\otimes k}=\Omega_{X}^{n}$ for $k>0$ and assume

1) No component of the base locus of $H^{\circ}(X, \mathscr{L})$ has codimension one.

2) $H_{\mathrm{Koz}}^{2}\left(H^{0}\left(X, \Omega^{n-1} \otimes \mathscr{L}^{i}\right)\right)=0$, for $-k \leqq i \leqq-1$.

Then the $n$ forms give local moduli, i.e. (1.1) is injective.

We will define the notion of $H_{\mathrm{Kox}}^{2}$ in $\S 2$. We note for the moment that if $H^{0}\left(X, \Omega^{n-1} \otimes \mathscr{L}\right)=0, \quad$ then $H^{0}\left(X, \Omega^{n-1} \otimes \mathscr{L}^{i}\right)=0 \quad$ for $i \leqq 1$. Indeed fixing $0 \neq s \in H^{\circ}(X, \mathscr{L})$, one has an injection $1 \otimes s^{(1-i)}: \Omega^{n-1} \otimes \mathscr{L}^{i} \rightarrow \Omega^{n-1} \otimes \mathscr{L}$ whence $H^{0}\left(X, \Omega^{n-1} \otimes \mathscr{L}^{i}\right)=0$. Thus the assumptions in Theorem 1 are strictly stronger than Theorem $1^{\prime}$.

In the case that $\mathscr{L}=\Omega_{X}^{n}$ and that the base locus has no components of codimension 2 , the new condition 2 is equivalent to the injectivity of (1.1). The group 
$H_{\mathrm{Koz}}^{2}\left(H^{0}\left(X, \Omega^{n-1} \otimes\left(\Omega^{n}\right)^{-1}\right)\right)$ gives a new method to recognize the failure of local Torelli. When $X$ is a curve, one may identify nonzero elements of $H_{\mathrm{Koz}}^{2}\left(H^{0}\left(X, \Omega^{n-1} \otimes\left(\Omega^{n}\right)^{-1}\right)\right)$ with nontrivial systems of quadratic equations vanishing on the canonical curve $f(X) \subseteq \mathbb{P}^{q-1}$. One may actually solve such a quadratic system to show that $f(X)$ is rational, i.e. that $X$ is hyperelliptic. One obtains therefore (cf. §3) an elementary proof of Noether's theorem.

\section{Proof of Theorem 1}

Given a compact complex manifold $X$, a line bundle $W$ and a subspace $0 \neq V \subseteq H^{0}(X, W)$ then we may form a vector bundle $E=W^{*} \otimes_{\mathbb{C}} V$. One has a canonical map $D: E \rightarrow \mathcal{O}_{X}$ sending $\varphi \otimes \sigma \rightarrow \varphi(\sigma)$. Extend this to form a Kozsul complex

$$
0 \rightarrow A^{q} E \rightarrow \ldots \rightarrow A^{2} E \rightarrow E \rightarrow \mathfrak{O} \rightarrow 0,
$$

where $D: A^{p} E \rightarrow A^{p+1} E$ satisfies

$$
D\left(\alpha_{1} \wedge \ldots \wedge \alpha_{p}\right)=\Sigma(-1)^{i} D\left(\alpha_{i}\right) \cdot\left(\alpha_{1} \wedge \ldots \wedge \hat{\alpha}_{i} \wedge \ldots \wedge \alpha_{p}\right) .
$$

Given any locally free $\mathcal{O}$-module $F$ we denote by $K_{W, V}^{\cdot}(F)=\operatorname{Hom}_{\mathcal{O}}(\Lambda E, F)$ the Kozsul complex of sheaves with differential $D^{*}$. When $W, V$ are understood from context we suppress the subscripts $W, V$. In particular if $V=H^{0}(X, W)$ we write simply $K_{W}(F)$.

Explicitly, if we pick a basis $x_{1}, \ldots, x_{g}$ for $V \subseteq H^{0}(X, W)$, and denote by $e_{1}, \ldots, e_{g}$ the dual basis for $V^{*}$ then identifying

$$
K^{p}(F)=\operatorname{Hom}_{\mathscr{O}}\left(\Lambda^{p} E, F\right)=F \otimes_{\mathcal{O}} \Lambda^{p} E^{*}=\left(F \otimes_{\mathcal{O}} W^{p}\right) \otimes_{\mathfrak{c}} \Lambda^{p} V^{*} .
$$

We may write sections of $K^{p}(F)$ as

$$
s=\sum_{i_{1}<\ldots<i_{p}} g_{i_{1} \ldots i_{p}} \cdot e_{i_{1}} \wedge \ldots \wedge e_{i_{p}},
$$

where $g_{i_{1} \ldots i_{p}}$ is a section of $F \otimes W^{p}$. The differential $D$ is

$$
D(s)=\sum_{i_{1}<\ldots<i_{p+1}}\left(\sum_{j}(-1)^{j_{x_{i}}} x_{i_{j}} g_{i_{1} \ldots \hat{i}_{j} \ldots i_{p}}\right) e_{i_{1}} \wedge \ldots \wedge e_{i_{p}},
$$

where $x_{i_{j}} \cdot g_{i_{1} \ldots i_{j} \ldots i_{p}}$ is a section of $F \otimes W^{p+1}$. For each fixed $q$ we obtain a "Kozsul" complex $K\left(H^{q}(X, F)\right)=H^{q}(X, K(F))$ where an element $s \in K^{p}\left(H^{q}(X, F)\right)$ has a representation as in (2.1) with the $g_{i_{1} \ldots i_{p}} \in H^{q}\left(X, W^{p} \otimes F\right)$ and $D$ is given by (2.2) with $x_{j} \cdot g_{i_{1} \ldots i_{p}} \in H^{q}\left(X, W^{p+1} \otimes F\right)$. We employ the notation

$$
\begin{aligned}
& H_{\mathrm{Koz} W V}^{p}\left(H^{q}(X, F)\right) \\
& =\frac{\operatorname{ker}\left\{H^{q}\left(X, F \otimes W^{p}\right) \otimes A^{p} V^{*} \rightarrow H^{q}\left(X, F \otimes W^{p+1}\right) \otimes A^{p+1} V^{*}\right\}}{\operatorname{im}\left\{H^{q}\left(X, F \otimes W^{p-1}\right) \otimes \Lambda^{p-1} V^{*} \rightarrow H^{q}\left(X, F \otimes W^{p}\right) \otimes A^{p} V^{*}\right\}} .
\end{aligned}
$$

The relevance of this construction to local Torelli is seen by taking $W=\Omega_{X}^{n}$, $V=H^{0}\left(X, \Omega_{X}^{n}\right), F=\Theta_{X}$ and noting that $H_{\mathrm{Koz}}^{0}\left(H^{1}(X, \Theta)\right)$ is precisely the kernel of the mapping $\lambda$ in (1.1), and our problem is to show this group vanishes.

For any $F, W, V$ we have an exact sequence

$$
0 \rightarrow H_{\mathbf{K}_{0 z}}^{1}\left(H^{0}(X, F)\right) \rightarrow H^{1} \rightarrow H_{\mathrm{K}_{0 z}}^{\mathrm{o}}\left(H^{\mathrm{l}}(X, F)\right) \rightarrow H_{\mathrm{K}_{0 \mathrm{z}}}^{2}\left(H^{0}(X, F)\right) \rightarrow \boldsymbol{H}^{2}
$$


of "initial terms" of the spectral sequence of hypercohomology ${ }^{1}$ of the complex of sheaves $K^{\dot{*}}(F)$. We claim [see (2.7)] that if $F$ is locally free and if the zero locus $Z$ of $x_{1}, \ldots, x_{g}$ has codimension $\geqq j$ then $\boldsymbol{H}^{k}=0$ for $k<j$, in particular

$$
0 \rightarrow H_{\mathbf{K}_{02}}^{0}\left(H^{1}(X, F)\right) \rightarrow H_{\mathbf{K}_{02}}^{2}\left(H^{0}(X, F)\right) \quad \text { if } \operatorname{cod} Z \geqq 2
$$

whence $H_{\mathrm{Koz}}^{0}\left(H^{1}\right)=0$ if $H_{\mathrm{Koz}}^{2}\left(H^{0}\right)=0$. (If and only if, when $\operatorname{cod} Z \geqq 3$.)

To obtain Theorem $1^{\prime}$ we apply these remarks with $W=L, V=H^{0}(X, W)$ and successively taking $F=\Theta \otimes L^{i}, i=0,1, \ldots, k-1$. We note that $\Theta \otimes \mathscr{L}^{i}$ $=\Theta \otimes \mathscr{L}^{k} \otimes \mathscr{L}^{i-k}=\Omega^{n-1} \otimes \mathscr{L}^{i-k}$ in view of the assumption $\mathscr{L}^{k}=\Omega^{n}$. Thus condition 2 of the theorem becomes $H_{\mathrm{K}_{0 z L} L}^{2}\left(H^{0}\left(X, \Theta \otimes \mathscr{L}^{i}\right)\right)=0, i=0, \ldots, k-1$, and hence

$$
H_{\mathrm{Koz} L}^{0}\left(H^{1}\left(X, \Theta \otimes \mathscr{L}^{i}\right)\right)=0
$$

for $i=0, \ldots, k-1$ in view of assumption 1 and (2.5) above. Now if local Torelli fails, there exists $0 \neq \alpha \in H^{1}(X, \Theta)$ such that $0=\sigma \cdot \alpha \in H^{1}\left(X, \Theta \otimes \mathscr{L}^{k}\right)$ for all $\sigma \in H^{0}\left(X, \mathscr{L}^{k}\right)$. Pick $1 \leqq j \leqq k$ minimal such that $\sigma \cdot \alpha=0$ for all $\sigma \in H^{0}\left(X, \mathscr{L}^{j}\right)$ and fix $\tau \in H^{0}\left(X, \mathscr{L}^{j-1}\right)$ such that $\tau \cdot \alpha \neq 0 \in H^{1}\left(X, \Theta \otimes \mathscr{L}^{j-1}\right)$. Then $\tau \cdot \alpha$ would define a nonzero element of $H_{\mathrm{K} 02 L}^{0}\left(H^{1}\left(X, \Theta \otimes \mathscr{L}^{j-1}\right)\right)$ contradicting our preceding analysis.

Remark 2.7. To complete the proof we need to see that $\boldsymbol{H}^{k}=0$ if $k<\operatorname{cod}(Z)$ and $F$ is locally free. From the second spectral sequence of hypercohomology

$$
H^{p}\left(X, \mathscr{K}^{q}\right) \Rightarrow H^{p+q}
$$

in which the $\mathscr{K}^{a}$ denote the cohomology sheaves of the complex $K^{\circ}(F)$, we see our assertion follows from the local assertion $\mathscr{K}^{q}=0$ if $q<\operatorname{cod} Z$, which is well known (see for example [3], p. 135). For the case of our particular interest $\operatorname{cod} Z>2$, the required assertion $\mathscr{K}^{0}=\mathscr{K}^{1}=0$ is quite elementary: Namely (1) if $x_{i} f=0$ for all $i$ and $f$ a section of $F$ then $f=0$ and (2) if $x_{i} f_{j}=x_{j} f_{i}$ for $f_{1}, \ldots, f_{g}$ sections of $F \otimes L$ then define a section of $F, g_{i}=f_{i} / x_{i}$ on $x_{i} \neq 0$ noting that $g_{i}=g_{j}$ on overlaps yielding a section of $F$ defined off $Z$, hence by Hartog's extension, a section $g$ of $F$ satisfying $x_{i} f=f_{i}$.

Remark 2.8. Peters' original proof of Theorem 1 was similar in spirit but employed his additional hypotheses to replace the spectral sequence argument with an argument having more geometric content. For example assuming $L$ imbeds $X$ in $\mathbb{P}^{N}$ one obtains an exact sequence

$$
0 \rightarrow \Theta_{X} \rightarrow \Theta_{\mathbf{p}^{N} \mid X} \rightarrow N \rightarrow 0
$$

and from the associated cohomology sequence

$$
0 \rightarrow H^{0}\left(\Theta_{X}\right) \rightarrow H^{0}\left(\Theta_{\left.\mathbf{p}^{\mathrm{n}}\right|_{X}}\right) \rightarrow H^{0}(N) \stackrel{\delta}{\rightarrow} H^{1}\left(\Theta_{X}\right) \rightarrow 0
$$

in which the surjectivity of $\delta$ is Peters' hypothesis 3 . He then worked globally employing arguments analogous to (2.7) to show that given $\alpha \in H^{\circ}(N)$ such that $x_{i} \cdot \delta(\alpha)=0$ for all $i$, there exists $\beta \in H^{0}\left(\Theta_{\mathbb{P N}_{\mid X}}\right)$ projecting to $\alpha$ hence $\delta(\alpha)=0$.

1 Fixing a good Cech covering $\mathscr{A}$ of $X$ one has a double complex $C^{q}\left(\mathfrak{A}, K^{p}(F)\right)$. The cohomology of the associated simple complex is denoted by $H^{\circ}$ and is called the hypercohomology of the complex $K(F)$. One studies $\boldsymbol{H}$ using the spectral sequences of the double complex 
Our present approach exposes an additional facet of the problem which deserves further exploration. Assuming that $\Omega_{X}^{n}$ is ample, (and hence so is $L$ ) we consider the singular Stein space $\hat{X}$ obtained by blowing down the zero section of $L^{*} \rightarrow X$. The sheaf $\pi^{*} \Theta_{X}$ on $\hat{X}-(0)$ extends canonically to a reflexive coherent sheaf $\hat{\Theta}$ on $\hat{X}$. (Namely, let $\tilde{\Theta}$ be the quotient of $\Theta_{X}$ by the vector field $V$ on $\hat{X}$ generated by the natural $\mathbb{C}^{*}$ action on $\hat{X}$ and let $\hat{\Theta}=\tilde{\Theta}^{* *}$.) The group $H^{0}\left(X, \Theta_{X}\right)$ is identifiable with the $\mathbb{C}^{*}$-invariants in $H^{0}(\hat{X}-\{0\}, \hat{\Theta})$. We view $H^{0}(\hat{X}-\{0\}, \hat{\Theta})$ as a graded module over the homogeneous coordinate ring $R=\operatorname{Sym}\left(H^{0}(X, \mathscr{L})\right)$ on $\mathbb{P}^{s^{-1}}$, $g=\operatorname{dim} H^{0}(X, \mathscr{L})$. One has the standard Kozsul complex

$$
0 \rightarrow \Lambda^{g} R^{(g)} \rightarrow \ldots \rightarrow \Lambda^{2} R^{(g)} \rightarrow R^{(g)} \rightarrow R \rightarrow 0
$$

associated to the $R$-sequence $x_{1}, \ldots, x_{g} \in R^{1}=H^{0}(X, \mathscr{L})$, which is an $R$-projective resolution of $\mathbb{C}$. Given any $R$ module $N$ the complex $K(N)=\operatorname{Hom}_{R}\left(\Lambda R^{(g)}, N\right)$, has as cohomology $\operatorname{Ext}_{R}^{i}(\mathbb{C}, N)$ and when $N$ is graded one obtains a natural grading on the Ext ${ }^{i}$. We easily identify our local Torelli obstructions $H_{\mathrm{Koz}}^{2}\left(H^{0}\left(X, \Theta_{X}\right)\right)$ with the degree 2 piece of $\operatorname{Ext}_{R}^{2}\left(\mathbb{C}, H^{0}(\hat{X}-\{0\}, \hat{\Theta})\right)$. Ideally one should be able to relate the nature of the isolated singularity at $0 \in \hat{X}$ to the failure of local Torelli. We note in this direction that if $\hat{X}$ is normal (i.e. $H_{f 0\}}^{i}\left(\hat{X}, \mathcal{O}_{\hat{X}}\right)=0$ for $i=0,1$ ), then $H^{0}(\hat{X}, \hat{\Theta}) \leadsto H^{0}(\hat{X}-\{0\}, \hat{\Theta})$ since $\hat{\Theta}$ is reflexive, cf. [6].

\section{Noether's Theorem}

Let $X$ be a nonsingular projective curve of genus $g=r+1$ and let $f: X \rightarrow \mathbb{P}^{r}$ be the canonical map. $X$ is called hyperelliptic if and only if $f(X)$ is a rational curve. (This occurs if and only if $f$ is not an imbedding.)

Theorem 2. The following are equivalent.

1) $X$ is hyperelliptic of genus $g \geqq 3$.

2) $H^{0}\left(X, \Omega_{X}^{1}\right)$ does not give local moduli.

3) The canonical map $\pi: H^{0}\left(X, \Omega_{X}^{1}\right) \otimes H^{0}\left(X, \Omega_{X}^{1}\right) \rightarrow H^{0}\left(X, \Omega_{X}^{\otimes 2}\right)$ is not surjective.

Proof. If 1) holds then representing $X$ as a 2 -sheeted cover of $\mathbb{P}^{1}$ one may explicitly calculate that $\pi$ in 3), is not surjective. The map $\pi$ is Serre dual to the map $\lambda$ of (1.1) and hence the surjectivity of $\pi$ is equivalent to the assertion 2). (See for example, [2], II.)

The result $2 \rightarrow 1$ or equivalently $3 \rightarrow 1$ is the classical Noether theorem, which we now demonstrate.

Let $x_{0}, \ldots, x_{r}$ be a basis for $H^{0}\left(X, \Omega^{1}\right)$. The failure of local Torelli is measured precisely by $H_{\mathbf{K} 0 \mathbf{z} \Omega^{1}}^{2}\left(H^{0}(X, \Theta)\right)$, i.e. by giving $\left(\begin{array}{l}g \\ 2\end{array}\right)$ elements $b_{i j} \in H^{0}\left(X, \Theta \otimes \Omega^{\otimes 2}\right), 0<i$ $<j \leqq r$ satisfying

$$
x_{i} b_{j k}-x_{j} b_{i k}+x_{k} b_{i j}=0 \in H^{0}\left(X, \Theta \otimes \Omega^{\otimes 3}\right)
$$

modulo collections of the form $b_{i j}=c_{j} x_{i}-c_{i} x_{j}$ for any choice of $c_{i} \in H^{0}\left(X, \Theta \otimes \Omega^{1}\right)$ $=H^{0}(X, \mathcal{O})=\mathbb{C}$. Note if $g=1$ or 2 then $\left(\begin{array}{l}g \\ 2\end{array}\right) \leqq 1$ and clearly $H_{\mathrm{Koz}}^{2}\left(H^{\mathrm{O}}(\Theta)\right)=0$. 
Assume $g \geqq 3$. Since $\operatorname{dim} X=1$ we have $\Theta \otimes \Omega^{\otimes n}=\Omega^{\otimes(n-1)}$ and we may view $b_{i j} \in H^{0}\left(X, \Omega^{1}\right)$ i.e. as a $\mathbb{C}$-linear combination of $x_{i}$ 's. Denote by $Q_{i j k}(x)=x_{i} b_{j k}-x_{j} b_{i k}$ $+x_{k} b_{i j} \in \operatorname{Sym}^{2}\left(H^{0}\left(X, \Omega^{1}\right)\right)$. Thus $Q_{i j k}$ is a quadratic polynomial in the $x_{i}$ which vanishes in $H^{\circ}\left(X, \Omega^{\otimes 2}\right)$, [i.e. $Q_{i j k}$ is a quadric vanishing on $f(X)$ ] in view of (2.1). However, vanishing of all the $Q_{i j k}$ as polynomials [i.e. in $\operatorname{Sym}^{2}\left(H^{0}\left(X, \Omega^{1}\right)\right.$ ] occurs if and only if $b_{i j}=c_{j} x_{i}-c_{i} x_{j}$, since the $x_{i}$ are an $R$-sequence in $\operatorname{Sym}\left(H^{0}\left(\Omega^{1}\right)\right)$. Thus the failure of local Torelli is equivalent to the existence of a nontrivial system of quadrics $Q_{i j k}$ vanishing on $f(X)$. We show that after a linear change of basis we can solve such a system of quadrics to express $x_{i} / x_{r} i=0, \ldots, r-2$ in terms of $x_{r-1} / x_{r}$. (If $g=3$ this is now obvious.)

We make the linear change of basis by selecting any non-Weierstrass point $p \in X$, and then choosing the basis $x_{0}, \ldots, x_{r}$ so that $v_{p}\left(x_{i}\right)=i$, where $v_{p}(f)$ denotes the order of zero of $f$ at $p$.

Lemma 2.2. We may normalize the choice of $\left\{b_{i j}\right\} i<j$ so that $v_{p}\left(b_{i j}\right) \geqq i+1$.

Proof. Every $b_{i j}$ is a linear combination of $x_{k}$ 's, and we shall say $b_{i j}$ "involves" $x_{k}$ if $x_{k}$ appears with nonzero coefficient. The assertion $v_{p}\left(b_{i j}\right) \geqq l$ is equivalent to the assertion $x_{k}$ not involved in $b_{i j}$ for $k<l$. We modify the $b_{i j}$ by a suitable boundary.

We claim that setting $c_{i}=$ Coefficient of $x_{0}$ in $b_{0 i}$ (and choosing any $c_{0}$ ) that the $b_{i j}^{\prime}=b_{i j}-\left(c_{j} x_{i}-c_{i} x_{j}\right)$ satisfy the lemma. Indeed

$$
x_{0} b_{i j}^{\prime}-x_{i} b_{0 j}^{\prime}+x_{j} b_{0 i}^{\prime}=0
$$

and since $x_{0}$ is not involved in $b_{0 k}^{\prime}$ for any $k$ we conclude that each of the last two summands has $v_{p} \geqq i+1$, whence $v_{p}\left(b_{i j}^{\prime}\right) \geqq i+1$ as asserted.

We remark that if $b_{r-1, r} \neq 0$ then we may now easily solve the equation since $x_{i} b_{r-1, r}=x_{r-1} b_{i r}-x_{r} b_{i r-1}$

and in view of our normalization the right hand side involves only $x_{k}$ with $k>i$ while $b_{r-1, r}=c \cdot x_{r}$.

We assume throughout the $b_{i j}$ are normalized, $v_{p}\left(b_{i j}\right)>i$.

Lemma 2.3. Fix i. Assume $b_{j, r}=0$ for all $j>i$. Then

a) $b_{i r}=d \cdot x_{r}$ for some $d$.

b) $b_{i j}=-d \cdot x_{j}$

c) $b_{j k}=0$ if $j>i$.

Proof. If $i=r-1$ there is nothing to prove, so assume $i<r-1$. From 2.1: $x_{r} b_{i, i+1}-x_{i} \cdot 0+x_{i+1} b_{i, r}=0$. Note that $v_{p}\left(x_{r} b_{i, i+1}\right) \geqq r+i+1$ whence $v_{p}\left(b_{i r}\right) \geqq r$ and a) follows. But also

$$
x_{r} b_{i j}-x_{i} \cdot 0+x_{j} b_{i r}=0
$$

whence setting $b_{i r}=d \cdot x_{r}$ we find $b$ ), [note that $f(X)$ is irreducible and not contained in a linear subspace of $\left.\mathbb{P}^{r}\right]$.

Part c) follows from the identity $x_{j} b_{k r}-x_{k} b_{j r}+x_{r} b_{j k}=0$.

Note that if $b_{j r}=0$ for all $j$ then $b_{j k} \equiv 0$ for all $j, k$. Thus given a non-cobounding cocycle there exists $i$ with $b_{i r} \neq 0$ and $b_{j r}=0 j>i$. [In fact $i>0$ since if $i=0$ one could modify by the coboundary $c_{j}=0 j>0$ and $c_{0}=$ coefficient of $x_{r}$ in $b_{0}$ obtaining a new normalized cocycle which is nesessarily 0 in view of (2.3).] Fix this $i$. 
Lemma 2.4. $b_{i-1, r}$ involves only $x_{r-1}, x_{r}$.

Proof. Since $b_{i r}=d x_{r}$ we see

$$
v_{p}\left(x_{i-1} b_{i, r}+x_{r} b_{i-1, i}\right)=r+i-1
$$

and hence $v_{p}\left(x_{i} b_{i-1, r}\right)=r+i-1$, whence $v_{p}\left(b_{i-1, r}\right)=r-1$ as asserted.

Now for $j<i$ one may employ

$$
x_{j} b_{i r}=x_{i} b_{j, r}-x_{r} b_{j, i}
$$

to solve for $x_{j} / x_{r}$ in terms of $x_{k} / x_{r}$ with $k>j$. Moreover for $i \leqq k<r-1$ one has

$$
x_{k+1} b_{i-1, r}=x_{r} b_{i-1, k+1} \text {. }
$$

Hence $v_{p}\left(x_{r} b_{i-1, k+1}\right)=k+1+r-1=k+r$ so that $v_{p}\left(b_{i-1, k+1}\right)=k$. Therefore $x_{k}$ (and no preceding $x_{l}$ ) appears in $b_{i-1, k+1}$ and one may use (2.5) to solve for $x_{k} / x_{r}$ in terms of $x_{l} / x_{r}$ with $l>k$. Thus recursively all the $x_{l} / x_{r}$ are expressed as rational functions of $x_{r-1} / x_{r}$, and consequently $f(X)$ is rational.

\section{References}

1. Bott, R.: Homogeneous vector bundles. Ann. Math. 66, 203-248 (1957)

2. Griffiths, $\mathrm{Ph}$ : Periods of integrals on algebraic manifolds. II. Amer. J. Math. 90, 805-865 (1968)

3. Matsumura,H.: Commutative algebra. New York: W.A.Benjamin 1970

4. Peters, C.: The local Torelli theorem. I. Complete intersections. Math. Ann. 217, 1-16 (1975); Erratum. Math. Ann. 223, 191-192 (1976)

5. Peters, C.: The local Torelli theorem. II. Cyclic branched coverings. Ann. Sc. Normale di Pisa, Ser. IV, 3, $321-340(1976)$

6. Schlessinger, M. : On rigid singularities. Rice Univers. Studies 59, $147-153$ (1973)

Received August 31, 1976, and in revised form January 30, 1977 